\title{
Meridional flow and differential rotation by gravity darkening in fast rotating solar-type stars
}

\author{
G. Rüdiger ${ }^{1}$ and M. Küker ${ }^{1,2}$ \\ 1 Astrophysikalisches Institut Potsdam, An der Sternwarte 16, 14482 Potsdam, Germany \\ 2 Astrophysikalisches Institut und Universitätssternwarte, Schillergässchen 2-3, 07745 Jena, Germany
}

Received 25 October 2001 / Accepted 10 January 2002

\begin{abstract}
An explanation is presented for the rather strong total surface differential rotation of the observed very young solar-type stars like AB Dor and PZ Tel. Due to its rapid rotation, a non-uniform energy flux leaves the stellar core so that the outer convection zone is non-uniformly heated from below. Due to this "gravity darkening" of the equator, a meridional flow is created flowing equatorwards at the surface and thus accelerating the equatorial rotation. The effect linearly grows with the normalized pole-equator difference, $\epsilon$, of the heatflux at the bottom of the convection zone. A rotation rate of about $9 \mathrm{~h}$ leads to $\epsilon=0.1$ for a solar-type star. In this case the resulting equator-pole differences of the angular velocity at the stellar surface, $\delta \Omega$, varies from unobservable 0.005 day $^{-1}$ to the (desired) value of 0.03 day $^{-1}$ when the dimensionless diffusivity factors $c_{\nu}$ and $c_{\chi}$ vary between 1 and 0.1 (standard value $c_{\nu} \simeq c_{\chi} \simeq 0.3$, see Table 1 ). In all cases the related temperature differences between pole and equator at the surface are unobservably small. The (clockwise) meridional circulation which we obtain flows opposite to the (counterclockwise) circulation appearing as a byproduct in the $\Lambda$-theory of the non-uniform rotation in outer convection zones. The consequences of this situation for those dynamo theories of stellar activity are discussed that work with the meridional circulation as the dominant magnetic-advection effect in latitude to produce the solar-like form of the butterfly diagram.
\end{abstract}

Key words. hydrodynamics - stars: rotation - stars: pre-main sequence - stars: activity

\section{Introduction}

In a series of papers, the internal rotation of outer convection zones of cool stars have been derived using a consistent mixing-length model of Reynolds stress and convective heat transport in a rotating convection zone that takes into account the effect of the Coriolis force on the convective motions (Küker et al. 1993; Kitchatinov \& Rüdiger 1995 (KR95); Kitchatinov \& Rüdiger 1999 (KR99)). In the KR95 model, the deviation of the heat flux from spherical symmetry causes a small horizontal temperature gradient, that partly neutralizes the rotational shear as the force that drives the meridional flow. As a result, the model reproduces the original Küker et al. (1993) result with only one free parameter, the mixing-length parameter $\alpha_{\mathrm{MLT}}$.

KR99 found the total shear,

$\delta \Omega=\Omega_{\mathrm{eq}}-\Omega_{\text {Pole }}$,

to be only rather weakly dependent on the rotation period, $\Omega$. Collier Cameron et al. (2001) have combined measurements of differential rotation for the pre-main-sequence

Send offprint requests to: G. Rüdiger,

e-mail: gruediger@aip.de stars RX J1508-4423, AB Dor, and PZ Tel with the sample of main-sequence stars from Donahue et al. (1996) and argue that the discrepancies between the KR99 result and the relation between shear and rotation rate derived by Donahue et al. (1996) may be due to the mix of G and K stars in the latter paper. They suggest that $\delta \Omega$ may indeed be constant with rotation rate but vary with spectral type and derive values for $\mathrm{G}$ and $\mathrm{K}$ dwarfs that differ by a factor of three, the $\mathrm{K}$ dwarfs rotating more rigidly. His discussion of the sample of photometric data for several classes of stars has led Hall (1991) to the surprisingly flat distribution profile $\delta \Omega \propto \Omega^{0.15}$. Further observations must decide how flat the $\delta \Omega-\Omega$ relation for solar-type stars really is.

In Fig. 1 the equator-pole differences (1) for the known rapid rotators are plotted in comparison to the results of the simulations by KR99. Obviously, the rapid rotators exhibit more differential rotation than the computations predict. One could believe - and we do - that another reason for differential rotation exists, which becomes important only for very rapid rotation.

In the present paper, the idea is adopted that for very fast rotation, a non-uniform energy flux leaves the stellar radiative core ("gravity darkening"), thus heating from 


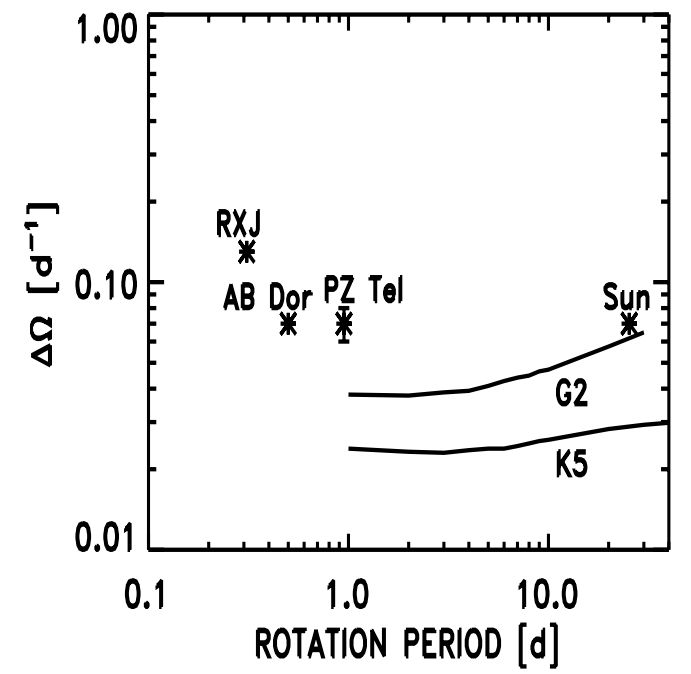

Fig. 1. The total equator-pole difference of the surface angular velocity (1) versus the period $\tau_{\text {rot }}$ of the basic rotation after the model in KR99 and as observed

below the stellar convection zone non-uniformly (Stẹpieǹ et al. 1997). In this way, a meridional circulation should arise in the convection zone which even by itself produces a non-uniform surface rotation (Kippenhahn 1963). We shall see that an accelerated equator is the natural consequence with an amplitude explaining indeed the surprisingly steep surface rotation laws which are followed by the fast rotators.

\section{The model}

In the diffusion approximation the radiative flux in a radiative stellar shell is proportional to the gradient of the temperature which itself is a function of the total gravitational potential $\phi=\psi-\int_{0}^{s} \Omega^{2} s^{\prime} \mathrm{d} s^{\prime}$. Hence

$\boldsymbol{F}=f(\phi) \nabla \phi=-f(\phi) \boldsymbol{g}^{\mathrm{eff}}$

with the effective gravity acceleration $\boldsymbol{g}^{\text {eff }}=\boldsymbol{g}-\Omega^{2} \boldsymbol{s}$. A convection zone above the radiative core is thus more intensely heated from below at the pole and it is less intensely heated from below at the equator. The intensity of this effect is given by the dimensional parameter

$\epsilon=\frac{\Omega^{2} R}{g}$.

Its value for solar-type stars is $\epsilon \simeq 0.014 /\left(\tau_{\text {rot }} / \text { day }\right)^{2}$ with $\tau_{\text {rot }}$ as the rotation period. For the very rapid rotation of 0.1 days the $\epsilon$ is of order unity and the star breaks off. In our computations the rotation parameter $\epsilon$ is considered as a free parameter; the models concern a star with a rotation period of 9 h, i.e. $\epsilon \simeq 0.1$. The same value would be true for a $\mathrm{K}$ giant with a 19 day rotation period. The K0 giant KU Peg, however, only has $\epsilon=0.04$ (see Weber \& Strassmeier 2001, for differential rotation and meridional flow $^{1}$ ).

\subsection{The transport equations}

We use the mean-field formulation of hydrodynamics, i.e. applying an appropriate averaging procedure to split the velocity field into a mean and a fluctuating part. The mean velocity field, $\overline{\boldsymbol{u}}$, is then governed by the Reynolds equation,

$\rho\left[\frac{\partial \overline{\boldsymbol{u}}}{\partial t}+(\overline{\boldsymbol{u}} \cdot \nabla) \overline{\boldsymbol{u}}\right]=-\nabla \cdot(\rho Q)-\nabla p+\rho \boldsymbol{g}$,

where $Q_{i j}=\left\langle u_{i}^{\prime} u_{j}^{\prime}\right\rangle$ is the correlation tensor of the velocity fluctuations, $\boldsymbol{u}^{\prime}$.

As the density distribution is spherically symmetric, and the gas motion is dominated by the global rotation, we assume axisymmetry for the mean velocity and temperature. The velocity field can then be described as a superposition of a rotation and a meridional flow, $\overline{\boldsymbol{u}}=r \sin \theta \Omega \hat{\boldsymbol{\phi}}+\overline{\boldsymbol{u}}^{\mathrm{m}}$, where $\hat{\boldsymbol{\phi}}$ is the unit vector in the azimuthal direction. The azimuthal component of the Reynolds equation expresses the conservation of angular momentum:

$\frac{\partial\left(\rho r^{2} \sin ^{2} \theta \Omega\right)}{\partial t}+\nabla \cdot \boldsymbol{t}=0$

where $\boldsymbol{t}=\rho r \sin \theta\left[r \sin \theta \Omega \overline{\boldsymbol{u}}^{\mathrm{m}}+\left\langle u_{\phi}^{\prime} \boldsymbol{u}^{\prime}\right\rangle\right]$. As the mass density varies with depth but not with time, mass conservation requires $\nabla \cdot(\rho \overline{\boldsymbol{u}})=0$. The meridional circulation can then be expressed by a stream function $A$. The stream function and the vorticity $\omega$ are related via the equation

$\Delta^{\prime} A-\frac{1}{\rho} \frac{\partial \rho}{\partial r} \frac{\partial A}{\partial r}-\frac{1}{\rho r} \frac{\partial \rho}{\partial r} A=-\rho \omega$,

with an operator $\Delta^{\prime}$ defined by $\Delta^{\prime}=\Delta-1 /\left(r^{2} \sin ^{2} \theta\right)$.

The correlation tensor $Q$ consists of a viscous and a non-viscous part. The eddy viscosity tensor,

$Q_{i j}^{\nu}=-\mathcal{N}_{i j k l} \frac{\partial \bar{u}_{k}}{\partial x_{l}}$

has been calculated by Kitchatinov et al. (1994). It is here simplified to $\mathcal{N}=\nu_{\mathrm{T}}\left(\delta_{i k} \delta_{j l}+\delta_{i l} \delta_{j k}\right)$ ignoring the effect of the basic rotation to the tensorial structure. For the eddy viscosity $\nu_{\mathrm{T}}$ the mixing-length expression

$\nu_{\mathrm{T}}=c_{\nu} \tau_{\mathrm{corr}} u_{\mathrm{T}}^{2}$

is used where, as usual, $\tau_{\text {corr }}$ is the convective turnover time and $u_{T}^{2}$ the turbulence intensity. The dimensionless parameter $c_{\nu}$ is model-dependent but it should not exceed unity. Its standard value is 0.3 but under the influence of rotation smaller values are also possible. The non-viscous $\Lambda$-effect is the main source of differential rotation. In order to select the influence of the gravity darkening of the

${ }^{1}$ Equatorial acceleration and poleward flow, quite similar to the Sun. 
equator, it is neglected, however, in the following calculations.

The convective heat transport is described by the transport equation

$\rho T \frac{\partial S}{\partial t}+\rho T \boldsymbol{u} \cdot \nabla S=-\nabla \cdot\left(\boldsymbol{F}^{\mathrm{conv}}+\boldsymbol{F}^{\mathrm{rad}}\right)+q$

where $q$ is the source function and $S$ the entropy. In the case of a perfectly adiabatic stratification the entropy is constant throughout the whole convection zone. Standard mixing-length theory does not include the rotational influence on the turbulent heat transport. Kitchatinov et al. (1994) have applied the same turbulence model as in their calculation of the Reynolds stress and found

$\boldsymbol{F}=\rho T \chi_{\mathrm{T}} \boldsymbol{\nabla} S$,

where

$\chi_{\mathrm{T}}=c_{\chi} \tau_{\mathrm{corr}} u_{\mathrm{T}}^{2}$

is the mixing-length expression for the heat conductivity coefficient in a slowly rotating convection zone, i.e. $\Omega^{*} \ll 1$. Again $c_{\chi}$ is a free parameter. More details of the corresponding thermodynamics are given by Küker \& Stix (2001).

\subsection{Model of the convection zone and boundary conditions}

KR95 used a simplified model of the convection zone which is derived from the full model by solving the equations

$\frac{\mathrm{d} T}{\mathrm{~d} r}=-\frac{g(r)}{C_{\mathrm{p}}}, \quad \frac{\mathrm{d} g}{\mathrm{~d} r}=-2 \frac{g}{r}+4 \pi G \rho, \quad \rho=\rho_{\mathrm{e}}\left(\frac{T}{T_{\mathrm{e}}}\right)^{\frac{1}{\gamma-1}}$

from a starting point $x_{\mathrm{e}}$, where the reference values $g_{\mathrm{e}}, \rho_{\mathrm{e}}$ and $T_{\mathrm{e}}$ are taken from a standard solar model. Together with the opacity law, $\kappa=0.34\left(1+6 \times 10^{24} \rho T^{-7 / 2}\right) \mathrm{cgs}$, this gives a stratification quite close to that of the model the reference values were taken from.

We additionally require that both the upper and lower boundaries are stress-free,

$Q_{r \phi}=Q_{r \theta}=0$.

As a boundary condition for the heat flux we require that the total flux through the outer boundary is equal to the total luminosity

$F_{r}^{\text {tot }}(r)=\frac{L}{4 \pi r^{2}}$

At the inner boundary the condition is now

$F_{r}^{\text {tot }}=\frac{L}{4 \pi r_{i}^{2}}\left(1+\frac{\epsilon}{\epsilon+3}\left(3 \cos ^{2} \theta-1\right)\right)$.

The parameter $\epsilon$ here gives the normalized difference between the heat flows at the poles and the equator,

$\epsilon=\frac{F_{r}^{\mathrm{tot}}(\text { pole })-F_{r}^{\mathrm{tot}}(\mathrm{eq})}{F_{r}^{\mathrm{tot}}(\mathrm{eq})}$

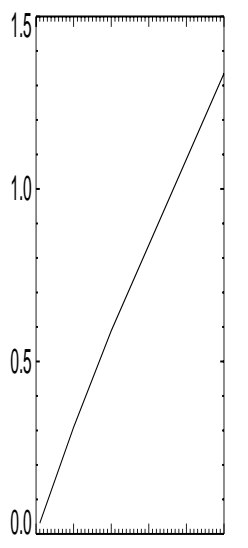

$\begin{array}{llllll}0.0 & 0.1 & 0.2 & 0.3 & 0.4 & 0.5\end{array}$ EPSLLON

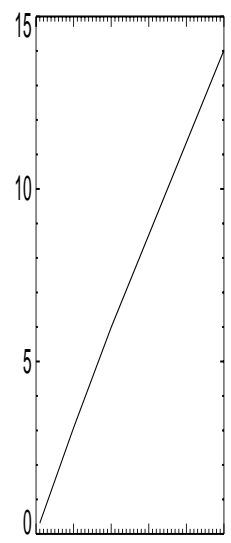

$\begin{array}{lllllll}0.0 & 0.1 & 0.2 & 0.3 & 0.4 & 0.5\end{array}$ EPSLLON

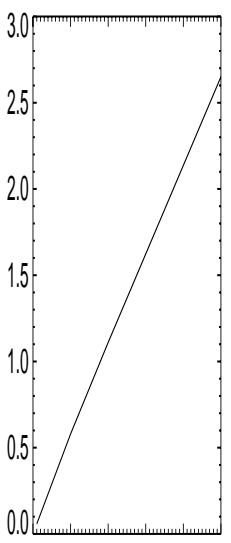

$\begin{array}{lllllll}0.0 & 0.1 & 0.2 & 0.3 & 0.4 & 0.5\end{array}$

EPSILON
Fig. 2. Left: the difference between the rotation rates at the equator and the the poles in deg/day. Middle: the maximum value of the (equatorward) meridional flow velocity at the surface in $\mathrm{ms}^{-1}$. Right: the maximum value of the (poleward) meridional flow velocity at the bottom in $\mathrm{ms}^{-1} \cdot c_{\nu}=c_{\chi}=1$

We have also tried the outer boundary condition of KR95,

$F_{r}^{\mathrm{tot}}\left(r_{\mathrm{e}}\right)=\frac{L}{4 \pi r_{\mathrm{e}}^{2}}\left(1+4 \frac{T \delta s}{C_{\mathrm{p}} T_{\mathrm{eff}}}\right)$

and found only small differences. However, (16) does not ensure that the luminosity is constant with radius and the solutions show a slow drift in the total entropy while with (13) a stationary state is reached after a few diffusion times.

\section{Results}

The results for $c_{\nu}=c_{\chi}=1$ are given in Fig. 2. We observe a nearly linear relation of the total equator-pole difference of the angular velocity and the amplitudes of the latitudinal drift at the bottom and the top of the convection zone with the darkening parameter $\epsilon$. We find for the equatorward flow

$u_{\text {top }} \simeq 31 \epsilon\left[\frac{\mathrm{m}}{\mathrm{s}}\right]$

and for the equator-pole difference

$\delta \Omega \simeq 0.058 \epsilon \quad\left[\right.$ day $\left.^{-1}\right]$,

both for small $\epsilon$. Both effects, of course, are negligible for the present-day Sun $\left(\epsilon \simeq 10^{-5}\right)$ but for rapid rotators with (say) $\epsilon \simeq 0.5$ the effects should be observable. With (3) for solar-type stars it follows

$\delta \Omega=7.9 \times 10^{-4} \frac{\text { day }}{\tau_{\text {rot }}^{2}}$.

For $\tau_{\text {rot }}=12 \mathrm{~h}$ one finds $\delta \Omega \simeq 0.003$ day $^{-1}$, a value which cannot explain the situation described in Fig. 1. For rotation periods exceeding 0.5 day and for the turbulence model used, the gravity darkening effect seems 

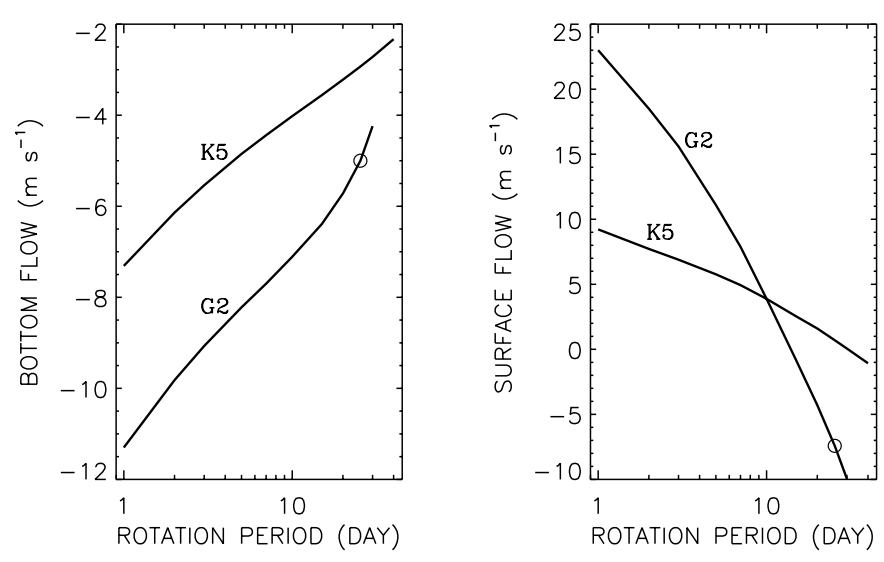

Fig. 3. The meridional circulation at mid-latitudes $\left(45^{\circ}\right)$ of the southern hemisphere at the bottom (left) and the top (right) of the convection zone for both a solar model and a K5 mainsequence star as a function of rotation period (KR99). The circle represents the present-day Sun. The flow at the bottom is equatorwards (as it is the flow at the solar surface, there are 2 cells in radius)

not to play an important role. As we shall see below, remarkably higher values for the equator-pole-difference are possible for modified turbulence parameters, but only up to a factor of 6 .

Another finding concerns the meridional flow. It has to do with the idea of interpreting the solar butterfly diagram as the result of a meridional circulation (equatorwards at the bottom of the convection zone) rather than as an intrinsic property of the $\alpha \Omega$-dynamo (Wang et al. 1991; Choudhuri et al. 1995; Dikpati \& Charbonneau 1999). To this end the eddy diffusivity in the convection zone must be much smaller than currently believed. A typical value of a suitable magnetic Prandtl number with which meridional flows of amplitude of $10 \mathrm{~ms}^{-1}$ become magnetohydrodynamically important is about 50 (Küker et al. 2001). The equatorward bottom drifts for stars with various rotation rates are given in Fig. 3. It varies from $5 \mathrm{~m} \mathrm{~s}^{-1}$ for the present-day Sun to $11 \mathrm{~m} \mathrm{~s}^{-1}$ for a very young solartype star. The bottom drift for the gravity darkening effect scales as

$u_{\mathrm{bot}} \simeq-5.8 \cdot \epsilon$

in $\mathrm{m} / \mathrm{s}$. It flows polewards, i.e. opposite to the meridional flow induced by the $\Lambda$-effect, without nonuniform heating from below. As a result of the nonuniform heating the influence of the meridional flow onto the migration of the stellar toroidal field belts is reduced. A new and interesting question is whether the total meridional flow can change its sign by the influence of nonuniform heating. The amplitude (20), however, seems to be too small to overcompensate $10 \mathrm{~m} \mathrm{~s}^{-1}$ even for the fastest rotation.

We have thus to vary the free eddy diffusion parameters $c_{\nu}$ and $c_{\chi}=c_{\nu} / \mathrm{Pr}$. The results for a variety of parameters $c_{\nu}$ and Pr are presented in Table 1 . In all cases they are written for $\epsilon=0.1$, i.e. for a rotation period of about
Table 1. Meridional flow at top and bottom of the convection zone, equator-pole differences of angular velocity and temperature for $\epsilon=0.1$ (rotation period of $\simeq 9 \mathrm{~h}$ ). Poleward circulation is marked with a minus, equatorward circulation with a plus.

\begin{tabular}{|l||c|c|c|c|c|}
\hline$c_{\nu}$ & 0.1 & 0.1 & 0.3 & 1 & 1 \\
$c_{\chi}$ & 0.1 & 0.3 & 0.3 & 1 & 0.3 \\
$\operatorname{Pr}$ & 1 & 0.3 & 1 & 1 & 3 \\
\hline$u_{\text {top }}\left[\mathrm{m} \mathrm{s}^{-1}\right]$ & +10 & +4.1 & +5.3 & +3.1 & +7.3 \\
$u_{\text {bot }}\left[\mathrm{m} \mathrm{s}^{-1}\right]$ & -1.3 & -0.46 & -0.81 & -0.58 & -1.50 \\
$\delta \Omega\left[\mathrm{day}^{-1}\right]$ & 0.03 & 0.013 & 0.012 & 0.005 & 0.010 \\
$\delta T[\mathrm{~K}]$ & 2 & 1 & 1 & 0.5 & 1 \\
\hline
\end{tabular}

$9 \mathrm{~h}$. There are no strong dependencies of the results on the input parameters $c_{\nu}$ and Pr. We have given in Table 1 also the heat conductivity parameter $c_{\chi}=c_{\nu} /$ Pr. Indeed, the equator-pole difference of the rotation rate has an inverse trend with $c_{\chi}$. For $c_{\chi}=1$ we find only $50 \%$ of $\delta \Omega$ resulting for $c_{\chi}=0.3$. For $c_{\chi}=0.1$ the factor of the corresponding $\delta \Omega$ is 6 , very close to the observations.

On the other hand, there is a clear trend of the meridional circulation at the bottom of the convection zone with the turbulent Prandtl number. When the Prandtl number grows by a factor of 10 the bottom drift increases by a factor of 3 . One can also find the reduction of the meridional flow for decreasing viscosity first described by Köhler (1969). His models demonstrate a distinct maximum of $10 \mathrm{~m} \mathrm{~s}^{-1}$ for an eddy viscosity of about $5 \times 10^{13} \mathrm{~cm}^{2} \mathrm{~s}^{-1}$. Density stratification, however, is not included. Models with density stratification require a faster meridional flow (a factor of 4 for a density contrast of 200) than models without density stratification (Rüdiger 1989, p. 113). For an eddy viscosity of $5 \times 10^{12}$ in a density-stratified convection zone, an equatorward circulation of $8 \mathrm{~m} \mathrm{~s}^{-1}$ at the surface produces an equator-pole difference of the angular velocity of $\delta \Omega \simeq 0.06 \mathrm{day}^{-1}$. The data given in the second column of Table 1 confirm this well-known result (see also Kippenhahn 1963).

In all cases the latitudinal differences of the temperature are unobservably small. The maximal value for $\epsilon=1$ is only $20 \mathrm{~K}$. This finding is in accordance with the finding that the convection zone is able to perform an effective screening of all large-scale temperature differences at the bottom of the convection zone (Stix 1981).

\section{References}

Choudhuri, A. R., Schüssler, M., \& Dikpati, M. 1995, A\&A, 303, L29

Collier Cameron, A., Barnes, J. R., \& Kitchatinov, L. L. 2001, in Cool Stars, Stellar Systems, and the Sun, 11, ed. R. J. Garcia Lopez, et al., ASP Conf. Ser., 223 
Dikpati, M., \& Charbonneau, P. 1999, ApJ, 518, 508

Donahue, R. A., Saar, S. H., \& Baliunas, S. L. 1996, ApJ, 466, 384

Hall, D. 1991, in The Sun and Cool Stars: Activity, magnetism, dynamos, ed. Tuominen et al., Lect. Notes in Phys., 380

Kippenhahn, R. 1963, ApJ, 137, 664

Kitchatinov, L. L., Pipin, V., \& Rüdiger, G. 1994, Astron. Nachr., 315, 157

Kitchatinov, L. L., \& Rüdiger, G. 1995, A\&A, 299, 446 (KR95)

Kitchatinov, L. L., \& Rüdiger, G. 1999, A\&A, 344, 911 (KR99)

Köhler, H. 1969, Differentielle Rotation als Folge anisotroper turbulenter Viskosität, Thesis, Göttingen
Küker, M., Rüdiger, G., \& Kitchatinov, L. L. 1993, A\&A, 279, L1

Küker, M., \& Stix, M. 2001, A\&A, 366, 668

Küker, M., Rüdiger, G., \& Schultz, M. 2001, A\&A, 374, 301

Rüdiger, G. 1989, Differential rotation and stellar convection: Sun and solar-type stars (Gordon \& Breach, New York)

Stępieǹ, K., Kiraga, M., Muthsam, K., \& Jahn, H. J. 1997, Acta Astron., 47, 235

Stix, M. 1981, A\&A, 93, 339

Wang, Y.-M., Sheeley, N. R., \& Nash, A. G. 1991, ApJ, 383, 431

Weber, M., \& Strassmeier, K. G. 2001, A\&A, 373, 974 